\title{
Approximate diagonals and Følner conditions for amenable group and semigroup algebras
}

\author{
by \\ Ross Stokke (Winnipeg)
}

\begin{abstract}
We study the relationship between the classical invariance properties of amenable locally compact groups $G$ and the approximate diagonals possessed by their associated group algebras $L^{1}(G)$. From the existence of a weak form of approximate diagonal for $L^{1}(G)$ we provide a direct proof that $G$ is amenable. Conversely, we give a formula for constructing a strong form of approximate diagonal for any amenable locally compact group. In particular we have a new proof of Johnson's Theorem: A locally compact group $G$ is amenable precisely when $L^{1}(G)$ is an amenable Banach algebra. Several structural $\mathrm{F} \varnothing$ lner-type conditions are derived, each of which is shown to correctly reflect the amenability of $L^{1}(G)$. We provide Følner conditions characterizing semigroups with 1-amenable semigroup algebras.
\end{abstract}

Introduction. Amenable Banach algebras were introduced by B. E. Johnson in [13] where he proved that a locally compact group $G$ is amenable if and only if its associated group algebra $L^{1}(G)$ is amenable (Johnson's Theorem). Shortly thereafter, Johnson proved his fundamental, intrinsic characterization of amenable Banach algebras in terms of the existence of virtual and approximate diagonals [14]. Virtual diagonals are often said to play the role in the theory of amenable Banach algebras that invariant means play in the theory of amenable groups.

In this paper we will study the exact relationship between the classical invariance properties possessed by amenable locally compact groups and the virtual/approximate diagonal "invariance properties" of their amenable group algebras. We will also address these matters in the context of a discrete semigroup algebra.

Amenable locally compact groups are characterized by a hierarchy of invariance properties, the deepest of which are the combinatorial Følner conditions. Følner conditions have also proven to be interesting and useful in the study of amenable semigroups, and are considered especially nice because

2000 Mathematics Subject Classification: 22D05, 22D15, 43A07, 43A20.

Key words and phrases: locally compact group, group algebra, semigroup algebra, amenability, approximate diagonal, virtual diagonal, Følner conditions. 
they describe amenability in terms of the internal structure of the group (or semigroup) itself, rather than in terms of an associated algebra [8], [18], [19], [20], [23]. We will show that amenable group algebras possess strong forms of approximate diagonals, thereby providing a corresponding hierarchy of approximate diagonals for amenable group algebras. In particular, we will establish Følner-type conditions characterizing discrete semigroups and locally compact groups whose associated $L^{1}$-algebras are 1-amenable. Thus one of our purposes here is to give internal properties of semigroups and locally compact groups which reflect the Banach algebra amenability of their associated $L^{1}$-algebras.

For a Banach algebra $A$ the projective tensor product $A \widehat{\otimes} A$ is a Banach $A$-bimodule with products determined by $(a \otimes b) \cdot c=a \otimes b c$ and $c \cdot(a \otimes b)=$ $c a \otimes b$. Let $\pi$ denote the multiplication operator determined by $\pi(a \otimes b)=a b$. An approximate diagonal for $A$ is a bounded net $\left(m^{\gamma}\right)$ in $A \widehat{\otimes} A$ such that for each $a \in A$,

$$
\lim _{\gamma}\left(m^{\gamma} \cdot a-a \cdot m^{\gamma}\right)=0, \quad \lim _{\gamma} \pi\left(m^{\gamma}\right) a=a .
$$

A virtual diagonal for $A$ is an element $M$ of the dual Banach $A$-bimodule $(A \widehat{\otimes} A)^{* *}$ such that for each $a \in A, M \cdot a=a \cdot M$ and $\left(\pi^{* *} M\right) a=a$. The Banach algebra $A$ is amenable if and only if it possesses an approximate diagonal, which is true if and only if it has a virtual diagonal [14]. The Banach algebra $A$ is called $k$-amenable, where $k$ is a positive constant, if it has an approximate (equivalently virtual) diagonal with bound $k$ (see [15]). Clearly $A$ is amenable precisely when it is $k$-amenable for some $k>0$. Proof of these statements and much more about amenable Banach algebras may be found for example in [3] and [21].

A classical result due to M. M. Day characterizes amenable locally compact groups $G$ in terms of nets $\left(f_{\alpha}\right)$ contained in $L^{1}(G)_{1}^{+}=\left\{f \in L^{1}(G)\right.$ : $f \geq 0$ and $\left.\|f\|_{1}=1\right\}$ which converge to invariance in $L^{1}(G)$, either with respect to translation by group elements or convolution by functions $f \in L^{1}(G)_{1}^{+}$. This latter sort of invariance is called topological invariance. Improving upon Day's result, Reiter's condition states that when $G$ is amenable the net $\left(f_{\alpha}\right)$ may be chosen so that

$$
\left\|\delta_{x} * f_{\alpha}-f_{\alpha}\right\|_{1} \rightarrow 0
$$

uniformly on compact subsets of $G$ (here $\delta_{x}$ denotes the Dirac measure at $x$ ). In fact amenable groups satisfy the Følner condition

(FC) For every $\varepsilon>0$ and every compact subset $K$ of $G$ there exists a compact subset $A$ of $G$ with $|A|>0$ such that

$$
|x A \triangle A|<\varepsilon|A| \quad \text { for every } x \in K
$$

(here $|B|$ denotes left Haar measure of the set $B$ and $\triangle$ denotes symmetric 
difference of sets). This says that the net $\left(f_{\alpha}\right)$ in $(*)$ may be taken to be comprised of normalized characteristic functions $\phi_{A}=|A|^{-1} 1_{A}, A \subset G$. Proofs of the statements made in this paragraph may be found in the monographs [8], [19], and [20].

A bounded approximate identity (bai) $\left(u_{\alpha}\right)$ for $L^{1}(G)$ is called quasicentral if

$$
\left\|\mu * u_{\alpha}-u_{\alpha} * \mu\right\|_{1} \rightarrow 0, \quad \mu \in M(G),
$$

where $M(G)$ is the measure algebra of $G$. As shown by V. Losert and H. Rindler, group algebras of amenable locally compact groups always have quasi-central bai's [17]. Further results on quasi-central bai's which are employed in this work are found in [22].

In Section 1, by use of Reiter's condition and the existence of a strong form of quasi-central bai for $L^{1}(G)$ (guaranteed by [17]), we provide an explicit formula for a strong form of approximate diagonal for $L^{1}(G)$ (Theorem 1.8). We show that weaker forms of approximate diagonals for $L^{1}(G)$ are sufficient for $G$ to be amenable (Proposition 1.2 and Corollary 1.3), and from the existence of an approximate diagonal of norm one, we show how to construct a net converging to topological invariance (Proposition 1.2). One immediate consequence is a new proof of Johnson's Theorem given entirely in terms of approximate diagonals.

Theorem 2.3 of Section 2 combines (FC) with a structural characterization of those unimodular groups whose group algebras have quasi-central bai's (from [22]) to obtain Følner-type conditions $\left(A_{\mathcal{V}}\right),\left(B_{\mathcal{V}}\right)$, and $\left(C_{\mathcal{V}}\right)$ for unimodular amenable groups. Theorem 2.5 shows that each of our properties $\left(\mathrm{A}_{\mathcal{V}}\right),\left(\mathrm{B}_{\mathcal{V}}\right)$, and $\left(\mathrm{C}_{\mathcal{V}}\right)$ is a "correct' Følner condition reflecting the amenability of $L^{1}(G)$ in the sense that each one yields a (compactly invariant) approximate diagonal for $L^{1}(G)$ comprised of normalized characteristic functions of subsets of $G \times G$.

It is well known that if $S$ is a semigroup, then amenability of the semigroup algebra $l^{1}(S)$ implies the amenability of $S$. However, unlike the case in which $S$ is a group, the converse does not hold. Thus a theme of several papers $([4],[5],[6],[9],[10]$ and portions of [16]) has been to address the problem of describing, in terms of the internal structure of the semigroup itself, those semigroups carrying amenable semigroup algebras. The general trend is that amenability of $l^{1}(S)$ imposes very strong conditions on $S$ (especially when combined with additional algebraic conditions placed upon the semigroup $S$ ). Indeed when $l^{1}(S)$ is amenable, it can be said that $S$ is "close" to a group. For a survey of what is known to date, see [11]. In Section 3, we show that for a discrete semigroup $S, l^{1}(S)$ is 1-amenable if and only if $S$ is an amenable group. The main result of this section provides three Følner-type conditions, one of which, with no extraneous conditions placed upon $S$, characterizes 1 -amenability of $l^{1}(S)$. 
This paper comprises a portion of the author's Ph.D. thesis, which was written under the supervision of Professor Anthony T.-M. Lau. The author wishes to express his deep gratitude to Professor Lau for his considerable encouragement and his valuable suggestions. The author would also like to thank Professors R. J. Loy and Y. Zhang for carefully reading and providing very useful comments upon earlier drafts of this paper. We further thank Professor Zhang for bringing the reference [24] to our attention. The financial support of the Natural Sciences and Engineering Research Council of Canada and the University of Alberta is gratefully acknowledged. We are grateful to the referee for some helpful comments regarding terminology.

1. Virtual and approximate diagonals for group algebras. In this section we begin our investigation of the relationship between the classical invariance properties of amenable locally compact groups and the approximate/virtual diagonals possessed by their associated amenable group algebras.

Throughout, $G$ will denote a locally compact group with left Haar measure $\lambda$, modular function $\Delta$, and identity $e$. For $a \in G$ and $f$ a function on $G$ we use the notation

$$
l_{a} f(t)=f(a t), \quad r_{a} f(t)=f(t a) \quad(t \in G) .
$$

Recall that $\lambda \times \lambda$ is a Haar measure, and $(x, y) \mapsto \Delta(x) \Delta(y),(x, y) \in G \times G$, is the associated modular function on $G \times G$. We will denote by $|A|$ the Haar measure of a Borel subset $A$ of either $G$ or $G \times G$. As usual, $L^{1}(G \times G)$ and $L^{1}(G) \widehat{\otimes} L^{1}(G)$ are identified through

$$
(h \otimes k)(s, t)=h(s) k(t) \quad\left(h, k \in L^{1}(G), s, t \in G\right) .
$$

References for Banach $G, L^{1}(G)$, and $M(G)$-modules are Chapter 2 of [13] and Section 11 of [20]. The following dual-module and $M(G)$-module operations are easily verified.

The canonical $L^{1}(G)$-module operations on $L^{1}(G \times G)$ extend to $M(G)$ through

$$
\mu \cdot m(s, t)=\int_{G} m\left(r^{-1} s, t\right) d \mu(r), \quad m \cdot \mu(s, t)=\int_{G} \Delta\left(r^{-1}\right) m\left(s, t r^{-1}\right) d \mu(r),
$$

where $m \in L^{1}(G \times G), \mu \in M(G)$. Thus $L^{1}(G \times G)$ has Banach $G$-module operations

$$
\begin{array}{r}
x \cdot m(s, t)=m\left(x^{-1} s, t\right), \quad m \cdot x(s, t)=\Delta\left(x^{-1}\right) m\left(s, t x^{-1}\right) \\
\left(m \in L^{1}(G \times G), x \in G\right) .
\end{array}
$$

Now

$$
x \cdot \phi=r_{(e, x)} \phi, \quad \phi \cdot x=l_{(x, e)} \phi \quad\left(x \in G, \phi \in L^{\infty}(G \times G)\right)
$$

describe the dual $G$-module operations on $L^{\infty}(G \times G)$. As usual the group 
algebra $L^{1}(G)$ is often viewed as a Banach $G$-module through the operations

$$
x \cdot f=\delta_{x} * f, \quad f \cdot x=f * \delta_{x} \quad\left(f \in L^{1}(G), x \in G\right) .
$$

The multiplication operator $\pi: L^{1}(G \times G) \rightarrow L^{1}(G)$ is given by

$$
\pi(m)(t)=\int_{G} m\left(s, s^{-1} t\right) d s \quad\left(m \in L^{1}(G \times G), s \in G\right),
$$

and we define $T: L^{1}(G \times G) \rightarrow L^{1}(G)$ through

$$
\operatorname{Tm}(s)=\int_{G} m(s, t) d t \quad\left(m \in L^{1}(G \times G), s \in G\right) .
$$

Observe that $T$ is a linear contraction. The following lemma contains some calculations from which the next two results will follow.

Lemma 1.1. (1) $\pi$ maps $L^{1}(G \times G)_{1}^{+}$into $L^{1}(G)_{1}^{+}$, and $\pi^{* *}$ maps the set of means on $L^{\infty}(G \times G)$ into the set of means on $L^{\infty}(G)$.

(2) If $\left(m^{\gamma}\right) \subset L^{1}(G \times G)$ is an approximate diagonal for $L^{1}(G)$, then

$$
\lim \left\langle 1_{G \times G}, m^{\gamma}\right\rangle=1 .
$$

(3) If $\mu \in M(G)$ and $m \in L^{1}(G \times G)$, then

$$
T(\mu \cdot m)=\mu *(T m), \quad T(m \cdot \mu)=\mu(G)(T m) .
$$

Proof. (1) is obvious.

(2) Let $\left(m^{\gamma}\right)$ be an approximate diagonal for $L^{1}(G)$. If $f \in L^{1}(G)_{1}^{+}$, then $1=\left\langle 1_{G}, f\right\rangle=\lim \left\langle 1_{G}, f * \pi\left(m^{\gamma}\right)\right\rangle=\left\langle 1_{G}, f\right\rangle\left\langle 1_{G}, \pi\left(m^{\gamma}\right)\right\rangle=\lim \left\langle 1_{G \times G}, m^{\gamma}\right\rangle$.

(3) For $m \in L^{1}(G \times G)$ and $s \in G$ we have

$$
\mu *(T m)(s)=\iint m\left(r^{-1} s, t\right) d t d \mu(r)=T(\mu \cdot m)(s)
$$

and

$$
T(m \cdot \mu)(s)=\iint \Delta\left(r^{-1}\right) m\left(s, t r^{-1}\right) d \mu(r) d t=\mu(G)(T m)(s) .
$$

We have the following link between approximate diagonals and nets converging to topological invariance.

Proposition 1.2. Let $G$ be a locally compact group. If there exists a bounded net $\left(m^{\gamma}\right) \subset L^{1}(G \times G)$ such that

$$
\lim \left\langle m^{\gamma}, 1_{G \times G}\right\rangle \neq 0, \quad\left\|f \cdot m^{\gamma}-m^{\gamma} \cdot f\right\|_{1} \rightarrow 0 \quad\left(f \in L^{1}(G)\right),
$$

then

$$
\left\|f * T m^{\gamma}-T m^{\gamma}\right\|_{1} \rightarrow 0 \quad\left(f \in L^{1}(G)_{1}^{+}\right),
$$

and $G$ is amenable. Hence, if $\left(m^{\gamma}\right) \subset L^{1}(G \times G)_{1}^{+}$, then $\left(T m^{\gamma}\right) \subset L^{1}(G)_{1}^{+}$ is a net converging to topological invariance.

Proof. Most of this follows from part (3) of Lemma 1.1. Let $n$ be a $w^{*}$-limit point of $\left(T m^{\gamma}\right)$; suppose without loss of generality that $T m^{\gamma} \stackrel{w^{*}}{\rightarrow} n$. 
Then for $\phi \in L^{\infty}(G)$ and $x \in G$ we have $n\left(l_{x} \phi\right)=n(\phi)$ (this follows from the topological invariance of $\left(\mathrm{Tm}^{\gamma}\right)$ as shown in [8, Proposition 2.1.3 and Corollary 2.4.4]). As well, $n\left(1_{G}\right)=\lim \left\langle 1_{G}, T m^{\gamma}\right\rangle=\lim \left\langle 1_{G \times G}, m^{\gamma}\right\rangle \neq 0$, so $n$ is non-zero. It follows that $G$ is amenable.

Observe that it follows from part (2) of Lemma 1.1 that any approximate diagonal for $L^{1}(G)$ satisfies the properties of the net $\left(m^{\gamma}\right)$ described in Proposition 1.2. The next statement lists some weaker forms of approximate and virtual diagonals which are sufficient for the amenability of $G$.

COROLlary 1.3. The following are equivalent for a locally compact group $G$ :

(1) $G$ is amenable.

(2) There exists a mean $M \in L^{\infty}(G \times G)^{*}$ such that $x \cdot M=M \cdot x$ $(x \in G)$.

(3) There exists a non-zero element $M \in L^{\infty}(G \times G)^{*}$ such that $x \cdot M=$ $M \cdot x(x \in G)$.

(4) There exists a net $\left(m^{\gamma}\right) \subset L^{1}(G \times G)_{1}^{+}$such that $\left\|x \cdot m^{\gamma}-m^{\gamma} \cdot x\right\|_{1} \rightarrow 0$ $(x \in G)$.

(5) There exists $M \in L^{\infty}(G \times G)^{*}$ such that $M\left(1_{G \times G}\right)=1$ and $f \cdot M=$ $M \cdot f\left(f \in L^{1}(G)\right)$.

(6) There exists a bounded net $\left(m^{\gamma}\right) \subset L^{1}(G \times G)$ such that

$$
\lim \left\langle m^{\gamma}, 1_{G \times G}\right\rangle=1, \quad\left\|f \cdot m^{\gamma}-m^{\gamma} \cdot f\right\|_{1} \rightarrow 0 \quad\left(f \in L^{1}(G)\right) .
$$

Proof. A stronger result than $(1) \Rightarrow(6)$ will be proved in Theorem 1.8, and $(6) \Rightarrow(1)$ follows from Proposition 1.2. The standard argument used to prove $[18$, Theorem 2.2] yields $(2) \Leftrightarrow(4)$ and $(5) \Leftrightarrow(6)$.

For $(1) \Leftrightarrow(2)$, note that if $G$ is amenable, then so is $G \times G$. Now take $M$ to be any two-sided invariant mean on $L^{\infty}(G \times G)$. For the converse, take $M$ as in condition (2) and let $n=T^{* *} M$. Then $n$ is a mean on $L^{\infty}(G)$, and from part (3) of Lemma 1.1, we have $x \cdot n=x \cdot\left(T^{* *} M\right)=T^{* *}(x \cdot M)=$ $T^{* *}(M \cdot x)=n$. That is, $n$ is a left invariant mean on $L^{\infty}(G)$.

Only $(3) \Rightarrow(2)$ remains. Taking $M$ as in (3), it is easy to see that we may suppose that $M$ is self-adjoint. Let $M$ have Jordan decomposition $M=M^{+}-M^{-}$. Now the $G$-module operations are isometric and preserve positivity in $L^{\infty}(G \times G)^{*}$, so it follows from the uniqueness of the Jordan decompositions of $x \cdot M$ and $M \cdot x$ that $x \cdot M^{+}=M^{+} \cdot x$ and $x \cdot M^{-}=M^{-} \cdot x$. If $M^{+} \neq 0$ (say) then

$$
M^{\prime}=\frac{1}{M^{+}\left(1_{G \times G)}\right.} M^{+}
$$

is a mean on $L^{\infty}(G \times G)$ such that $x \cdot M^{\prime}=M^{\prime} \cdot x$.

REMARKs 1.4. Corollary 1.3 also holds for any discrete semigroup. In this situation, $(2) \Rightarrow(1)$ is [4, Lemma 3], and for inverse semigroups $(1) \Rightarrow(2)$ 
is [4, Lemma 4]. If $S$ has an identity, our argument above proves $(1) \Rightarrow(2)$, however for an arbitrary semigroup, the implication may still be obtained using an argument similar to that of $[12,17.18(b)]$.

We will now show that amenable group algebras have strong forms of approximate diagonals, and we will describe their construction from the amenability properties of the underlying group. We require some facts about quasi-central bounded approximate identities due to V. Losert and H. Rindler [17].

A net $\left(e_{\beta}\right)$ in $L^{1}(G)$ is called quasi-central if $\left\|\mu * e_{\beta}-e_{\beta} * \mu\right\|_{1} \rightarrow 0$ $(\mu \in M(G))$. A mean $m$ on $L^{\infty}(G)$ is called inner invariant if $m\left(x \cdot \phi \cdot x^{-1}\right)$ $=m(\phi)\left(\phi \in L^{\infty}(G), x \in G\right)$, and is called an extension of the Dirac measure $\delta_{e}$ (from $\mathrm{CB}(G)$ to $\left.L^{\infty}(G)\right)$ if $m(\phi)=\phi(e)(\phi \in \mathrm{CB}(G)$ ). Contained in [17, Theorem 5] is the fact that $L^{1}(G)$ has a quasi-central bounded approximate identity if and only if $L^{\infty}(G)$ has an inner invariant mean which extends the Dirac measure at $e$. The following are respectively [17, Theorem 3] and [17, Theorem 2].

TheOREM 1.5 (Losert and Rindler). Let $G$ be a locally compact group.

(1) If $G$ is amenable, then $L^{1}(G)$ has a quasi-central bai.

(2) Suppose that $L^{1}(G)$ has a quasi-central bai $\left(e_{\beta}\right)$. Then $\left(e_{\beta}\right)$ may be chosen so that $\left(e_{\beta}\right) \subset L^{1}(G)_{1}^{+}$and $\left\|\delta_{x} * e_{\beta}-e_{\beta} * \delta_{x}\right\|_{1} \rightarrow 0$ uniformly on compact subsets of $G$.

We note that alternate proofs of the above statements may be found in [22]. Moreover, it is shown in [22] that when $L^{1}(G)$ has a quasi-central bai $\left(e_{\beta}\right)$, then it may be chosen so that both properties from Theorem 1.5(2) are satisfied, and for any neighbourhood $U$ of $e$, there exists $\beta_{0}$ such that $\operatorname{support}\left(e_{\beta}\right) \subset U$ whenever $\beta \succeq \beta_{0}$. Applications of this extension of [17, Theorem 2] are found in [22].

DeFinition 1.6. We will say that an approximate diagonal $\left(m^{\gamma}\right) \subset$ $L^{1}(G \times G)_{1}^{+}$is compactly invariant [respectively measure invariant] if

$$
\left\|x \cdot m^{\gamma}-m^{\gamma} \cdot x\right\|_{1} \rightarrow 0
$$

uniformly on compact subsets of $G$ [respectively $\left\|\mu \cdot m^{\gamma}-m^{\gamma} \cdot \mu\right\|_{1} \rightarrow 0$ $(\mu \in M(G))]$. A virtual diagonal $M$ for $L^{1}(G)$ is measure invariant if $M$ is a mean and $\mu \cdot M=M \cdot \mu(\mu \in M(G))$.

The following proposition contains some simple observations.

Proposition 1.7. Let $G$ be a locally compact group.

(1) Let $\left(m^{\gamma}\right)$ be an approximate diagonal for $L^{1}(G)$. If $\left(m^{\gamma}\right)$ is compactly invariant, then $\left(m^{\gamma}\right)$ is measure invariant, and $\left(\pi\left(m^{\gamma}\right)\right)$ is a quasicentral bai contained in $L^{1}(G)_{1}^{+}$such that $\left\|\delta_{x} * \pi\left(m^{\gamma}\right)-\pi\left(m^{\gamma}\right) * \delta_{x}\right\|_{1}$ $\rightarrow 0$ uniformly on compact subsets of $G$. If $\left(m^{\gamma}\right)$ is measure invari- 
ant, then $\left(\pi\left(m^{\gamma}\right)\right)$ is a quasi-central bounded approximate identity in $L^{1}(G)_{1}^{+}$.

(2) Let $M$ be a measure invariant virtual diagonal for $L^{1}(G)$. Then $\pi^{* *} M$ is an inner invariant mean on $L^{\infty}(G)$ which extends the Dirac measure at $e$.

Proof. (1) Let $\left(m^{\gamma}\right)$ be a compactly invariant approximate diagonal for $L^{1}(G)$. For $\mu \in M(G)$ with compact support we have

$$
\begin{aligned}
\left\|\mu \cdot m^{\gamma}-m^{\gamma} \cdot \mu\right\|_{1} & =\iint\left|\int m^{\gamma}\left(r^{-1} s, t\right)-\Delta\left(r^{-1}\right) m^{\gamma}\left(s, t r^{-1}\right) d \mu(r)\right| d s d t \\
& \leq \int\left\|r \cdot m^{\gamma}-m^{\gamma} \cdot r\right\|_{1} d|\mu|(r),
\end{aligned}
$$

which converges to zero. The balance of (1) now follows from Lemma 1.1 and the fact that $\pi$ is an $M(G)$-module morphism.

(2) The fact that $\pi^{* *}(M)$ is a mean is from Lemma 1.1. Now $\pi^{* *}$ is an $M(G)$-module morphism so $\pi^{* *}(M)$ is inner invariant; as $\pi^{* *}(M)$ is a weak*-limit point of a bounded approximate identity, it extends the Dirac measure at $e$ (see [17]).

We remark that on page 319 of [2] it is incorrectly stated that when $G$ is an amenable locally compact group, one can construct a virtual diagonal for $L^{1}(G)$ as follows: letting $m$ be an invariant mean on $L^{\infty}(G)$, define

$$
M(h)=\int_{G} h\left(t, t^{-1}\right) d m(t) \quad\left(h \in L^{\infty}(G \times G)\right),
$$

where the formal integral represents the action of $m$ on the function $t \mapsto$ $h\left(t, t^{-1}\right)$. However unless $G$ is discrete, the set $\left\{\left(t, t^{-1}\right): t \in G\right\}$ may have measure zero so the map $M$ is not well defined. We have only chosen to mention this as further motivation for this study, and we wish to stress that this one false statement does not affect the validity of any of the results in [2]. Indeed, the author would like to thank Professor Galé for pointing out that he and his co-author only require their map $M$ for continuous bounded functions and not all of $L^{\infty}(G \times G)$. One must only notice that the $\pi$-invariance which is used in [2] to characterize the amenability of $G$ refers to $\pi(G)$-invariance.

THEOREM 1.8. Let $G$ be an amenable locally compact group. Let $\left(f_{\alpha}\right) \subset$ $L^{1}(G)_{1}^{+}$be a net such that $\left\|\delta_{x} * f_{\alpha}-f_{\alpha}\right\|_{1} \rightarrow 0$ uniformly on compact subsets of $G$. Let $\left(e_{\beta}\right) \subset L^{1}(G)_{1}^{+}$be a quasi-central bai for $L^{1}(G)$ as in Theorem 1.5(2). For each $\gamma=(\alpha, \beta)$, define

$$
m^{\gamma}(s, t)=f_{\alpha}(s) e_{\beta}(s t), \quad(s, t) \in G \times G .
$$

Then $\left(m^{\gamma}\right)$ is a compactly invariant approximate diagonal for $L^{1}(G)$ contained in $L^{1}(G \times G)_{1}^{+}$. 
Proof. It is easy to see that $\left(m^{\gamma}\right) \subset L^{1}(G \times G)_{1}^{+}$. Observe that for any $x \in G$ and any $(s, t) \in G \times G$,

$$
\begin{aligned}
& x \cdot m^{\gamma}(s, t)=m^{\gamma}\left(x^{-1} s, t\right)=\delta_{x} * f_{\alpha}(s) \delta_{x} * e_{\beta}(s t), \\
& m^{\gamma} \cdot x(s, t)=\Delta\left(x^{-1}\right) m^{\gamma}\left(s, t x^{-1}\right)=f_{\alpha}(s) e_{\beta} * \delta_{x}(s t) .
\end{aligned}
$$

Therefore

$$
\begin{aligned}
\left\|x \cdot m^{\gamma}-m^{\gamma} \cdot x\right\|_{1}= & \iint\left|\delta_{x} * f_{\alpha}(s) \delta_{x} * e_{\beta}(s t)-f_{\alpha}(s) e_{\beta} * \delta_{x}(s t)\right| d t d s \\
= & \iint\left|\delta_{x} * f_{\alpha}(s) \delta_{x} * e_{\beta}(t)-f_{\alpha}(s) e_{\beta} * \delta_{x}(t)\right| d t d s \\
\leq & \iint\left|\delta_{x} * f_{\alpha}(s)-f_{\alpha}(s)\right| \delta_{x} * e_{\beta}(t) d t d s \\
& \quad+\iint f_{\alpha}(s)\left|\delta_{x} * e_{\beta}(t)-e_{\beta} * \delta_{x}(t)\right| d t d s \\
= & \left\|\delta_{x} * f_{\alpha}-f_{\alpha}\right\|_{1}+\left\|\delta_{x} * e_{\beta}-e_{\beta} * \delta_{x}\right\|_{1} \rightarrow 0
\end{aligned}
$$

uniformly on compact subsets of $G$. A calculation shows that for any $\gamma=$ $(\alpha, \beta)$, and any $s \in G, \pi\left(m^{\gamma}\right)(s)=e_{\beta}(s)$. Thus $\left(\pi\left(m^{\gamma}\right)\right)$ is a (quasi-central) bai for $L^{1}(G)$.

REMARKS 1.9. Suppose conversely that $\left(m^{\gamma}\right)$ is a compactly invariant approximate diagonal for $L^{1}(G)$. From Lemma $1.1(3)$, we have $\left(T m^{\gamma}\right) \subset$ $L^{1}(G)_{1}^{+}$and $\left\|\delta_{x} * T m^{\gamma}-T m^{\gamma}\right\|_{1} \rightarrow 0$ uniformly on compact subsets of $G$. This is Reiter's condition. Moreover, by Proposition 1.7, $\left(\pi\left(m^{\gamma}\right)\right) \subset L^{1}(G)_{1}^{+}$ is a quasi-central bai for $L^{1}(G)$ such that $\left\|\delta_{x} * \pi\left(m^{\gamma}\right)-\pi\left(m^{\gamma}\right) * \delta_{x}\right\|_{1} \rightarrow 0$ uniformly on compact subsets of $G$.

One immediate consequence of this work is a new proof of Johnson's Theorem given entirely in terms of approximate diagonals.

COROLlaRY 1.10 (Johnson's Theorem). The following are equivalent for a locally compact group $G$ :

(1) $G$ is amenable.

(2) $L^{1}(G)$ is 1-amenable.

(3) $L^{1}(G)$ is amenable.

Proof. $(1) \Rightarrow(2)$ is contained in Theorem 1.8 and $(2) \Rightarrow(3)$ is trivial. $(3) \Rightarrow(1)$ follows from Lemma 1.1(2) and Proposition 1.2.

In the language of Definition 1.6 we have proved

COROLlary 1.11. The following are equivalent for a locally compact group $G$ :

(1) $G$ is amenable.

(2) $L^{1}(G)$ has a compactly invariant approximate diagonal.

(3) $L^{1}(G)$ has a measure invariant approximate diagonal.

(4) $L^{1}(G)$ has a measure invariant virtual diagonal. 
In [24], Yong Zhang studies Banach algebras $A$ which have approximate diagonals $\left(m^{\gamma}\right)$ satisfying

$$
a \cdot m^{\gamma}=m^{\gamma} \cdot a \quad(a \in A) .
$$

He shows that group algebras of compact groups always have approximate diagonals with this property [24, Proposition 6]. We note here that this is the only case in which a group algebra can have an approximate diagonal of this form.

Proposition 1.12. Let $G$ be a locally compact group. Then $L^{1}(G)$ has an approximate diagonal satisfying condition (1) if and only if $G$ is compact.

Proof. If $L^{1}(G)$ has an approximate diagonal $\left(m^{\gamma}\right)$ satisfying (1), then the proof of Proposition 1.2 shows that for some $\gamma_{0}, \mathrm{Tm}^{\gamma_{0}} \neq 0$ and $\delta_{x} * T m^{\gamma_{0}}=T m^{\gamma_{0}}$ for every $x \in G$. Thus $\operatorname{Tm}^{\gamma_{0}} \in L^{1}(G)$ is equal almost everywhere to a non-zero constant, and it follows that $G$ is compact. The converse, which is proved in [24], is a special case of our Theorem 1.8: let $m^{\beta}(s, t)=e_{\beta}(s t)$ where $\left(e_{\beta}\right)$ is a central bai for $L^{1}(G)$.

REMARKS 1.13. In this section we have examined the relationship between nets converging to invariance, quasi-central bai's and approximate diagonals for $L^{1}(G)$. Wendel's theorem tells us that $M(G)$ is the multiplier algebra of $L^{1}(G)$. For any Banach algebra $A$, there are natural analogues of quasi-central bai's, nets converging to (probability measure) invariance, and measure invariant approximate diagonals which can be stated in terms of the associated multiplier algebra $\mathcal{M}(A)$. Both nets converging to invariance and quasi-central bai's are clearly less complicated than approximate diagonals and have the advantage that they can be studied separately. It would thus be interesting to see what can be said along these lines in the context of other amenable Banach algebras.

2. Følner conditions. In this section we will derive Følner-type conditions for amenable group algebras. We will employ the Følner condition (FC) and a structural condition from [22] which characterizes those unimodular groups whose group algebras have quasi-central bai's. We show that all of our conditions are correct Følner conditions reflecting the amenability of $L^{1}(G)$ in the sense that they yield (compactly invariant) approximate diagonals for $L^{1}(G)$ comprised of normalized characteristic functions.

For any $C \subset G$, we use the notation

$$
\nabla(C)=\{(x, y): x y \in C\},
$$

and for $A \subset G \times G, x \in G$ we write

$$
x \cdot A=\{(x s, t):(s, t) \in A\}, \quad A \cdot x=\{(s, t x):(s, t) \in A\} .
$$


The normalized characteristic function $|A|^{-1} 1_{A}$ of a Borel measurable subset $A$ with positive measure is denoted by $\phi_{A}$.

Let $\mathcal{V}$ be any base for the neighbourhood system at $e$. In the following definitions of conditions $\left(\mathrm{A}_{\mathcal{V}}\right),\left(\mathrm{B}_{\mathcal{V}}\right)$, and $\left(\mathrm{C}_{\mathcal{V}}\right)$, the subsets $A$ of $G \times G$ and $K$ of $G$ are to be assumed compact and of positive measure.

$\left(\mathrm{A}_{\mathcal{V}}\right) \quad$ For any $\varepsilon>0, K \subset G$, and $V \in \mathcal{V}$, there exists $A \subset G \times G$ such that

(i) $A \subset \nabla(V)$,

(ii) $|x \cdot A \triangle A \cdot x|<\varepsilon|A|(x \in K)$.

$(\mathrm{B} \mathcal{V}) \quad$ For any $\varepsilon>0, K \subset G$, and $V \in \mathcal{V}$, there exists $A \subset G \times G$ such that

(i) $|A \backslash \nabla(V)|<\varepsilon|A|$,

(ii) $|x \cdot A \triangle A \cdot x|<\varepsilon|A|(x \in K)$.

$\left(\mathrm{C}_{\mathcal{V}}\right) \quad$ For any $\varepsilon>0, K \subset G$, and $V \in \mathcal{V}$, there exists $A \subset G \times G$ such that

(i) $|A \cap \nabla(V)|>(1-\varepsilon)|A|$,

(ii) $|x \cdot A \cap A \cdot x|>(1-\varepsilon)|A|(x \in K)$.

Conditions (i) of $\left(\mathrm{A}_{\mathcal{V}}\right),\left(\mathrm{B}_{\mathcal{V}}\right)$ and $\left(\mathrm{C}_{\mathcal{V}}\right)$ say that the sets $A$ lie very close to the reverse-diagonal $\nabla(e)=\left\{\left(x, x^{-1}\right): x \in G\right\}$.

LEMMA 2.1. Suppose that there exists a net $\left(A_{\alpha}\right)$ of measurable subsets of $G \times G$ with $0<\left|A_{\alpha}\right|<\infty$ such that

$$
\frac{\left|x \cdot A_{\alpha} \triangle A_{\alpha} \cdot x\right|}{\left|A_{\alpha}\right|} \rightarrow 0 \quad(x \in G) .
$$

Then $G$ is unimodular and amenable.

Proof. For each $x \in G$ and each $\alpha$,

$$
\begin{aligned}
\Delta(x) & =\frac{\left|A_{\alpha} \cdot x\right|}{\left|A_{\alpha}\right|}=\frac{1}{\left|A_{\alpha}\right|}\left[\left|A_{\alpha} \cdot x \backslash x \cdot A_{\alpha}\right|+\left|x \cdot A_{\alpha}\right|-\left|x \cdot A_{\alpha} \backslash A_{\alpha} \cdot x\right|\right] \\
& =1+\frac{\left|A_{\alpha} \cdot x \backslash x \cdot A_{\alpha}\right|}{\left|A_{\alpha}\right|}-\frac{\left|x \cdot A_{\alpha} \backslash A_{\alpha} \cdot x\right|}{\left|A_{\alpha}\right|} .
\end{aligned}
$$

Taking the limit of the final term of the above equation we obtain $\Delta(x)=1$ for each $x \in G$. Now the net $\left(\phi_{A_{\alpha}}\right)$ satisfies

$$
\left\|x \cdot \phi_{A_{\alpha}}-\phi_{A_{\alpha}} \cdot x\right\|_{1}=\frac{\left|x \cdot A_{\alpha} \triangle A_{\alpha} \cdot x\right|}{\left|A_{\alpha}\right|},
$$

which converges to zero. Thus $G$ is amenable by Corollary 1.3.

The next result is proved by use of the Følner condition (FC) and the following which is [22, Theorem 3.3]. 
THEOREM 2.2. The following are equivalent for a locally compact group $G$ :

(1) $G$ is unimodular and $L^{1}(G)$ has a quasi-central bounded approximate identity.

(2) There exists a net $\mathcal{U}=\left(U_{\alpha}\right)$ comprising a base for the neighbourhood system at e such that

$$
\frac{\left|x U_{\alpha} \triangle U_{\alpha} x\right|}{\left|U_{\alpha}\right|} \rightarrow 0
$$

uniformly on compact subsets of $G$. The sets $U_{\alpha}$ may be chosen to be compact and symmetric.

THEOREM 2.3. Let $G$ be a locally compact group, and let $\mathcal{V}$ be any base for the neighbourhood system at e. The following are equivalent:

(1) $G$ is amenable and unimodular.

(2) $G$ satisfies condition $\left(\mathrm{A}_{\mathcal{V}}\right)$.

(3) $G$ satisfies condition $(\mathrm{B} \mathcal{V})$.

(4) $G$ satisfies condition $\left(\mathrm{C}_{\mathcal{V}}\right)$.

Proof. Suppose that $G$ satisfies condition (Bv). If for each pair $\alpha=(\varepsilon, K)$, we choose a corresponding subset $A_{\alpha}$ of $G \times G$ as in condition (ii) of (BV), then by Lemma $2.1, G$ is amenable and unimodular. Thus $(3) \Rightarrow(1)$, and similarly $(4) \Rightarrow(1)$. Now $(3) \Leftrightarrow(4)$ follows from the identity $|x \cdot A \triangle A \cdot x|=$ $2(|A|-|x \cdot A \cap A \cdot x|)$, and $(2) \Rightarrow(3)$ is clear. Only $(1) \Rightarrow(2)$ remains.

Let $0<k<1, K \subset G$ compact, and $V \in \mathcal{V}$. It is sufficient to find a compact set $A$ such that $A \subset \nabla(V)$ and $|x \cdot A \cap A \cdot x|>k|A|(x \in K)$. By use of condition (FC), Theorem 1.5(1), and Theorem 2.2 we can find compact subsets $B$ and $U$ of $G$ with $U$ a neighbourhood of $e$ contained in $V$ such that

$$
|x B \cap B|>\sqrt{k}|B|, \quad|x U \cap U x|>\sqrt{k}|U| \quad(x \in K) .
$$

Let $A=\{(s, t): s \in B$ and $s t \in U\}$. Obviously $A \subset \nabla(V)$. Now it is easy to see that for any $x \in G, x \cdot A=\{(s, t): s \in x B$ and $s t \in x U\}$, and $A \cdot x=\{(s, t): s \in B$ and $s t \in U x\}$. It follows that $x \cdot A \cap A \cdot x=\{(s, t)$ : $s \in x B \cap B$ and $s t \in x U \cap U x\}$. But if $C$ and $W$ are any two measurable subsets of $G$ with finite measure, and $E=\{(s, t): s \in C$ and $s t \in W\}$, then

$$
\begin{aligned}
|E| & =\iint 1_{E}(x, y) d y d x=\iint 1_{E}\left(x, x^{-1} y\right) d y d x \\
& =\iint 1_{C \times W}(x, y) d y d x=|C||W| .
\end{aligned}
$$

Thus for any $x \in K$,

$$
|x \cdot A \cap A \cdot x|=|x B \cap B||x U \cap U x|>\sqrt{k}|B| \sqrt{k}|U|=k|A| .
$$

In order to show that our Følner conditions reflect the amenability of $L^{1}(G)$, we will need the following lemma. We note that unimodularity is not required. 
Lemma 2.4. Let $G$ be a locally compact group, and let $A$ be a Borel measurable subset of $G \times G$ with $0<|A|<\infty$. For any Borel measurable subset $U$ of $G$ with $|U|<\infty$ we have

$$
\left\|\pi\left(\phi_{A}\right) * 1_{U}-1_{U}\right\|_{1}=\frac{2}{|A|} \int_{U}\left|A \backslash \nabla\left(x U^{-1}\right)\right| d x .
$$

Proof. For any $x \in G$ we have

$$
\begin{aligned}
\pi\left(1_{A}\right) * 1_{U}(x) & =\iint 1_{A}\left(s, s^{-1} t\right) 1_{U}\left(t^{-1} x\right) d s d t \\
& =\iint 1_{A}(s, t) 1_{U}\left(t^{-1} s^{-1} x\right) d t d s=\left|A \cap \nabla\left(x U^{-1}\right)\right| .
\end{aligned}
$$

It follows that

$$
\begin{aligned}
\int_{G \backslash U} \pi\left(1_{A}\right) * 1_{U}(x) d x & =\int_{G} \pi\left(1_{A}\right) * 1_{U}(x) d x-\int_{U} \pi\left(1_{A}\right) * 1_{U}(x) d x \\
& =\int_{U}|A| d x-\int_{U}\left|A \cap \nabla\left(x U^{-1}\right)\right| d x \\
& =\int_{U}\left|A \backslash \nabla\left(x U^{-1}\right)\right| d x .
\end{aligned}
$$

We now obtain

$$
\begin{aligned}
\left\|\pi\left(\phi_{A}\right) * 1_{U}-1_{U}\right\|_{1} & =\int_{U}\left|\pi\left(\phi_{A}\right) * 1_{U}(x)-1\right| d x+\int_{G \backslash U} \pi\left(\phi_{A}\right) * 1_{U}(x) d x \\
& =\frac{1}{|A|}\left[\int_{U}|| A \cap \nabla\left(x U^{-1}\right)|-| A|| d x+\int_{U}\left|A \backslash \nabla\left(x U^{-1}\right)\right| d x\right] \\
& =\frac{2}{|A|} \int_{U}\left|A \backslash \nabla\left(x U^{-1}\right)\right| d x .
\end{aligned}
$$

Suppose that $G$ is an amenable and unimodular locally compact group and let $\mathcal{V}$ be any base for the neighbourhood system at $e$. Let

$$
\mathcal{T}_{\mathcal{V}}=\{\gamma=(\varepsilon, K, V): \varepsilon>0, K \subset G \text { compact with }|K|>0, V \in \mathcal{V}\}
$$

and direct $\mathcal{T}_{\mathcal{V}}$ by putting

$$
\gamma_{0}=\left(\varepsilon_{0}, K_{0}, V_{0}\right) \preceq \gamma_{1}=\left(\varepsilon_{1}, K_{1}, V_{1}\right) \Leftrightarrow \varepsilon_{1} \leq \varepsilon_{0}, K_{1} \supset K_{0}, V_{1} \subset V_{0} .
$$

For each $\gamma=(\varepsilon, K, V) \in \mathcal{T}_{\mathcal{V}}$, take a subset $A_{\gamma}$ of $G \times G$ to correspond to $(\varepsilon, K, V)$ as in any one of the conditions $\left(\mathrm{A}_{\mathcal{V}}\right),\left(\mathrm{B}_{\mathcal{V}}\right)$ or $\left(\mathrm{C}_{\mathcal{V}}\right)$.

TheOREM 2.5. Let $G$ be a unimodular amenable locally compact group. The nets $\left(\phi_{A_{\gamma}}\right)$ of normalized characteristic functions are compactly invariant approximate diagonals for $L^{1}(G)$.

Proof. For notational convenience we write $\phi_{\gamma}=\phi_{A_{\gamma}}$. Observe that in all cases we have 


$$
\left\|x \cdot \phi_{\gamma}-\phi_{\gamma} \cdot x\right\|=\frac{\left|x \cdot A_{\gamma} \triangle A_{\gamma} \cdot x\right|}{\left|A_{\gamma}\right|} \rightarrow 0
$$

uniformly on compact subsets of $G$.

We must show that the nets $\left(\pi\left(\phi_{\gamma}\right)\right)$ are bounded approximate identities for $L^{1}(G)$. This will follow if we can show that for any compact subset $L_{0}$ of $G$ with $\left|L_{0}\right|>0,\left\|\pi\left(\phi_{\gamma}\right) * 1_{L_{0}}-1_{L_{0}}\right\|_{1} \rightarrow 0$. We do this in the case that the sets $A_{\gamma}$ are chosen with respect to condition $\left(\mathrm{B}_{\mathcal{V}}\right)$. To this end, let $\varepsilon_{0}>0$, and take an open neighbourhood $U$ of $L_{0}$ such that $\left|U \backslash L_{0}\right|<\varepsilon_{0} / 6$. Take a neighbourhood $V_{0} \in \mathcal{V}$ such that $V_{0}^{-1} L_{0} \subset U$ and suppose that $\gamma=(\varepsilon, K, V) \succeq \gamma_{0}=\left(\varepsilon_{0} / 6\left|L_{0}\right|, K_{0}, V_{0}\right)$. Then $V \subset x U^{-1}$ for each $x \in L_{0}$, and so

$$
\left|A_{\gamma} \backslash \nabla\left(x U^{-1}\right)\right| \leq\left|A_{\gamma} \backslash \nabla(V)\right|<\varepsilon\left|A_{\gamma}\right| \quad\left(x \in L_{0}\right) .
$$

Using this and Lemma 2.4 we obtain

$$
\begin{aligned}
\left\|\pi\left(\phi_{\gamma}\right) * 1_{L_{0}}-1_{L_{0}}\right\|_{1} & =\left\|\pi\left(\phi_{\gamma}\right) *\left(1_{U}-1_{U \backslash L_{0}}\right)-\left(1_{U}-1_{U \backslash L_{0}}\right)\right\|_{1} \\
& \leq\left\|\pi\left(\phi_{\gamma}\right) * 1_{U}-1_{U}\right\|_{1}+\left\|\pi\left(\phi_{\gamma}\right)\right\|_{1}\left\|1_{U \backslash L_{0}}\right\|_{1}+\left\|1_{U \backslash L_{0}}\right\|_{1} \\
& \leq \int_{U} 2 \frac{\left|A_{\gamma} \backslash \nabla\left(x U^{-1}\right)\right|}{\left|A_{\gamma}\right|} d x+\frac{\varepsilon_{0}}{3} \\
& \leq \int_{U \backslash L_{0}} 2 d x+\int_{L_{0}} 2 \varepsilon d x+\frac{\varepsilon_{0}}{3} \\
& \leq \frac{2 \varepsilon_{0}}{3}+2 \varepsilon\left|L_{0}\right| \leq \varepsilon_{0} .
\end{aligned}
$$

This argument also works when the sets $A_{\gamma}$ are chosen to correspond to $\left(\mathrm{A}_{\mathcal{V}}\right)$ or $\left(\mathrm{C}_{\mathcal{V}}\right)$. Note however that when the sets $A_{\gamma}$ correspond to $\left(\mathrm{A}_{\mathcal{V}}\right)$, each function $\pi\left(\phi_{\gamma}\right) \in L^{1}(G)_{1}^{+}$is supported on $V$, and it is immediate that $\left(\pi\left(\phi_{\gamma}\right)\right)$ is a bounded approximate identity for $L^{1}(G)$.

REMARKS 2.6. (1) Let $G$ be any unimodular locally compact group which is amenable. Let $\mathcal{U}=\left(U_{\delta}\right)$ be a base for the neighbourhood system at $e$ as in Theorem 2.2 and let $\left(K_{\gamma}\right)$ be a Følner net for $G$ in the sense that it satisfies condition (3) of [19, Definition 4.15]. Let

$$
A_{\gamma, \delta}=\left\{(s, t): s \in K_{\gamma} \text { and } s t \in U_{\delta}\right\} .
$$

Then the argument used in the proof of Theorem 2.3 together with Theorem 2.5 shows that the net $\left(\phi_{A_{\gamma, \delta}}\right)$ is a compactly invariant approximate diagonal for $L^{1}(G)$. If $G$ is $\sigma$-compact and first countable (that is, metrizable), then this approximate diagonal may be chosen to be a sequence. We remark that much work has been done regarding the explicit construction of Følner nets for certain classes of locally compact groups [19, Chapter 6]. It would be interesting if for such groups one could similarly construct asymp- 
totically invariant nets as described in Theorem 2.2. If so, we would have a method for explicitly constructing compactly invariant approximate diagonals, comprised of normalized characteristic functions, for group algebras of such groups. When $G$ is abelian, any base $\mathcal{U}$ for the neighbourhood system at $e$ satisfies condition (2) of Theorem 2.2 so it is particularly easy to construct approximate diagonals for $L^{1}(G)$. For example, if $G$ is the additive group of real numbers, letting $A_{n}=\{(s, t): s \in[-n, n]$ and $s+t \in[-1 / 4 n, 1 / 4 n]\}$, the sequence of characteristic functions $\left(1_{A_{n}}\right)$ is a compactly invariant approximate diagonal for $L^{1}(\mathbb{R})$.

(2) The Følner condition (FC) was proved for discrete groups by E. Følner in [7]. An elegant proof due to I. Namioka appears in [18]. Employing Namioka's method, (FC) is proved in [8] for amenable locally compact groups by use of Reiter's condition $[8,3.6 .2]$. We note that one can interpret condition (2) of Corollary 1.11 as a Reiter condition for amenable group algebras. Doing this, based upon Namioka's method and our Lemma 2.4, one can directly derive other Følner conditions for amenable group algebras. Indeed in the author's Ph.D. thesis (University of Alberta, 2003), a $F \varnothing$ lner condition $\left(\mathrm{F}_{\mathcal{V}}\right)$, corresponding to a base for the neighbourhood system $\mathcal{V}$ of $e$, is derived in this manner. It is also shown to correctly reflect the amenability of $L^{1}(G)$ in the sense of Theorem 2.5. The condition is as follows:

$(\mathrm{F} \mathcal{V}) \quad$ For every $\varepsilon>0$, every $\delta>0$, every compact $K \subset G$, and every $V \in \mathcal{V}$, there exists a compact set $A \subset G \times G$ and $N \subset V$ with $|A|>0$ and $|N|<\delta$ such that

(i) $\left|A \backslash \nabla\left(x V^{-1}\right)\right|<\varepsilon|A|(x \in V \backslash N)$,

(ii) $|x \cdot A \triangle A \cdot x|<\varepsilon|A|(x \in K)$.

(3) In the introduction to this paper we listed a hierarchy of nets converging to invariance characterizing amenable locally compact groups. We have now provided an analogous hierarchy of approximate diagonals for amenable group algebras.

3. 1-Amenability of semigroup algebras. Throughout this final section, $S$ will denote a (discrete) semigroup. In the context of our semigroup $S$, we will consider the natural analogues of the Følner conditions from the previous section. The conditions - (A), (B), and (C) - are shown to exactly correspond to 1-amenability of $l^{1}(S)$. We will also observe that with no extraneous conditions placed upon $S$, if the semigroup algebra $l^{1}(S)$ is 1-amenable, then $S$ is necessarily an amenable group.

If $E$ is a subset of $S,|E|$ is its cardinality. We write

$$
\left[s^{-1} t\right]=\{x \in S: s x=t\}, \quad\left[s t^{-1}\right]=\{x \in S: s=x t\} \quad(s, t \in S) .
$$


As in the case for groups, we may identify $l^{1}(S) \widehat{\otimes} l^{1}(S)$ with $l^{1}(S \times S)$ and we shall identify the Dirac function at an element $s$ of $S$ with $s$ itself. Doing this, a function $m^{\gamma} \in l^{1}(S \times S)$ may be written in the form

$$
m^{\gamma}=\sum_{S \times S} \beta_{s, t}^{\gamma}(s, t)
$$

and one sees that the net $\left(\mathrm{m}^{\gamma}\right)$ is an approximate diagonal for $l^{1}(S)$ if and only if it is bounded, and for each $v \in S$,

$$
\lim _{\gamma} \sum_{S \times S} \beta_{s, t}^{\gamma}[(s, t v)-(v s, t)]=0, \quad \lim _{\gamma} \sum_{S \times S} \beta_{s, t}^{\gamma} s t v=v .
$$

Further details regarding these identifications may be found in [4].

Definition 3.1. The semigroup $S$ is left cancellative if $\left|\left[s^{-1} t\right]\right| \leq 1$ for any $s, t \in S$. If for any $s \in S$, $\sup \left\{\left|\left[s^{-1} t\right]\right|: t \in S\right\}<\infty$, then $S$ will be called left subcancellative.

Recall from [16] that $S$ is left weakly cancellative if $\left|\left[s^{-1} t\right]\right|<\infty$ for any $s, t \in S$. It is clear that left cancellativity implies left subcancellativity, which in turn implies left weak cancellativity. That these implications cannot be reversed is fairly easy to show.

For $A \subset S \times S$ and $v \in S, v \cdot A, A \cdot v$ are as defined in Section 2, and we write

$$
\nabla(v)=\{(s, t) \in S \times S: s t v=v\} .
$$

In the following definitions of Følner-type conditions (A), (B), and (C), the subsets $A$ of $S \times S$ and $F$ of $S$ are to be assumed finite and non-empty:

(A) For any $\varepsilon>0, F \subset S$, there exists $A \subset S \times S$ such that

$$
A \subset \nabla(v), \quad|v \cdot A \triangle A \cdot v|<\varepsilon|A| \quad(v \in F) .
$$

(B) For any $\varepsilon>0, F \subset S$, there exists $A \subset S \times S$ such that

$$
|A \backslash \nabla(v)|+|v \cdot A \triangle A \cdot v|<\varepsilon|A| \quad(v \in F) .
$$

(C) For any $\varepsilon>0, F \subset S$ there exists $A \subset S \times S$ such that

$$
|A \cap \nabla(v)|+|v \cdot A \cap A \cdot v|>(2-\varepsilon)|A| \quad(v \in F) .
$$

We note that when $S$ is a group, for any $v \in S$ we have $\nabla(v)=\left\{\left(s, s^{-1}\right)\right.$ : $s \in S\}$, so the conditions $\left(\mathrm{A}_{\mathcal{V}}\right),\left(\mathrm{B}_{\mathcal{V}}\right)$, and $\left(\mathrm{C}_{\mathcal{V}}\right)$ of Section 2 are non-discrete analogues of these new conditions.

THEOREM 3.2. The following are equivalent for a semigroup $S$ :

(1) $l^{1}(S)$ is 1-amenable.

(2) $S$ is an amenable group.

(3) $S$ is one-sided subcancellative, and satisfies condition (A).

(4) $S$ is one-sided subcancellative, and satisfies condition (B).

(5) $S$ satisfies condition (C). 
EXAMPLE 3.3. Let $S$ be the semigroup of positive integers with multiplication given by $n \cdot m=\min \{n, m\}$. Then $S$ satisfies condition (A) (and therefore condition (B)), yet $S$ is not a group. To see this let $\varepsilon>0$, and let $F$ be a finite subset of $S$. Let $m=\max (F)$, and put $B=\{m+1, \ldots, m+n\}$, where $2 / n<\varepsilon$. Then for any $v \in F, A=B \times B \subset \nabla(v)$, and

$$
|v \cdot A \triangle A \cdot v| /|A|=(|v \cdot A|+|A \cdot v|) /|A|=2 n / n^{2}<\varepsilon .
$$

This example shows that at least some form of cancellativity is needed in conditions (3) and (4) of the theorem. Note however that our example is not even weakly cancellative (for any $n \in S,\left[n^{-1} n\right]$ is infinite). It would be interesting to find an example of a weakly cancellative semigroup which is not a group, yet satisfies (A).

To prove the theorem we need some preliminary results.

Lemma 3.4. If $v \in S$, and $\sup \left\{\left|\left[v^{-1} t\right]\right|: t \in S\right\} \leq n$, then for any finite $A \subset S \times S,|v \cdot A| \geq n^{-1}|A|$.

Proof. Write $A$ as $A=\bigcup_{i=1}^{m}\left\{x_{i}\right\} \times C_{i}$, where $x_{i} \neq x_{j}$ whenever $i \neq j$. Let $B=\left\{x_{1}, \ldots, x_{m}\right\}, v B=\left\{y_{1}, \ldots, y_{k}\right\}$, and for $1 \leq j \leq k$, let $M_{j}=$ $\left\{i: v x_{i}=y_{j}\right\}$. Then $\left|M_{j}\right| \leq n$, and

$$
v \cdot A=\bigcup_{i=1}^{m}\left\{v x_{i}\right\} \times C_{i}=\bigcup_{j=1}^{k}\left(\left\{y_{j}\right\} \times \bigcup_{i \in M_{j}} C_{i}\right) .
$$

Thus

$$
|v \cdot A|=\sum_{j=1}^{k}\left|\bigcup_{i \in M_{j}} C_{i}\right| \geq \sum_{j=1}^{k} \frac{1}{\left|M_{j}\right|} \sum_{i \in M_{j}}\left|C_{i}\right| \geq \frac{1}{n} \sum_{j=1}^{k} \sum_{i \in M_{j}}\left|C_{i}\right|=\frac{1}{n}|A| .
$$

A right simple semigroup which contains an idempotent is called a right group. A discussion of right groups can be found in $\S 1.11$ of [1].

Lemma 3.5. A semigroup $S$ is a right group if and only if

(†) for any $v_{1}, v_{2} \in S$ (not necessarily distinct), there exists $(s, t) \in \nabla\left(v_{1}\right)$ such that $s \in v_{2} S$.

Proof. Assume that $S$ satisfies ( $\dagger$ ) and suppose that $I$ is a (non-empty) proper right ideal in $S$. Take $v_{1} \in S \backslash I, v_{2} \in I$ and choose $(s, t) \in \nabla\left(v_{1}\right)$ such that $s \in v_{2} S$. Then $s \in I$, so $v_{1}=s t v_{1} \in I$, a contradiction. Thus $S$ is right simple. It now suffices to exhibit an idempotent in $S$. To this end, let $v \in S$ and take $(s, t) \in \nabla(v)$ with $s=v x$ for some $x \in S$. Then $(x t v)^{2}=(x t)(v x) t v=(x t)(s t v)=x t v$. The converse (which we do not need) follows easily from the fact that right groups are regular and right simple. 
LEMMA 3.6. If $S$ satisfies condition (C), or if $S$ is left subcancellative and satisfies condition (B), then $S$ is a right group.

Proof. Suppose first that $S$ is left subcancellative and satisfies condition (B). We will show that $S$ satisfies condition ( $\dagger$ ) of Lemma 3.5. Let $v_{1}, v_{2} \in S$ and suppose that $\sup \left\{\left|\left[v_{2}^{-1} t\right]\right|: t \in S\right\} \leq n$. Take $A$ to be a finite non-empty subset of $S \times S$ such that

$$
\left|A \backslash \nabla\left(v_{i}\right)\right|+\left|v_{i} \cdot A \triangle A \cdot v_{i}\right|<\frac{1}{2 n}|A| \quad(i=1,2) .
$$

Then using Lemma 3.4 we have

$\frac{1}{2 n}|A|>\left|v_{2} \cdot A \backslash A \cdot v_{2}\right|=\left|v_{2} \cdot A\right|-\left|v_{2} \cdot A \cap A \cdot v_{2}\right| \geq \frac{1}{n}|A|-\left|v_{2} \cdot A \cap A \cdot v_{2}\right|$, and so $\left|v_{2} \cdot A \cap A \cdot v_{2}\right|>(2 n)^{-1}|A|$. Suppose that for every $(s, t) \in A \cap \nabla\left(v_{1}\right)$, $\left(s, t v_{2}\right) \notin v_{2} \cdot A$. Then $v_{2} \cdot A \cap A \cdot v_{2} \subset\left\{\left(s, t v_{2}\right):(s, t) \in A \backslash \nabla\left(v_{1}\right)\right\}$, so

$$
\frac{1}{2 n}|A|<\left|v_{2} \cdot A \cap A \cdot v_{2}\right| \leq\left|A \backslash \nabla\left(v_{1}\right)\right|<\frac{1}{2 n}|A|,
$$

a contradiction. Thus for some $(s, t) \in A \cap \nabla\left(v_{1}\right),\left(s, t v_{2}\right) \in v_{2} \cdot A$; in particular $(s, t) \in \nabla\left(v_{1}\right)$, and $s \in v_{2} S$. To see that condition (C) implies that property $(\dagger)$ holds is similar, but easier: arguing as above let $\varepsilon=1 / 2$ in condition (C) applied to $v_{1}, v_{2} \in S$. Then $\left|v_{2} \cdot A \cap A \cdot v_{2}\right|>2^{-1}|A|$ and $\left|A \backslash \nabla\left(v_{1}\right)\right|<2^{-1}|A|$, so the preceding argument completes the proof.

If $A$ is a finite subset of $S \times S$, let $\phi_{A}$ denote the normalized characteristic function $|A|^{-1} 1_{A}$ of $A$.

LEMMA 3.7. Let $S$ be a left cancellative semigroup which satisfies the following condition in which the sets $F$ and $A$ are to be assumed finite and non-empty:

(*) For any $\varepsilon>0, F \subset S$, there exists $A \subset S \times S$ such that

$$
|A \backslash \nabla(v)|+|v \cdot A \backslash A \cdot v|<\varepsilon|A| \quad(v \in F) .
$$

Then $l^{1}(S)$ has an approximate diagonal comprised of normalized characteristic functions of finite non-empty subsets of $S \times S$.

Proof. Fix $A \subset S \times S, v \in S$. If for $(x, y) \in S \times S$ we write $\left[(x, y) \cdot v^{-1}\right]$ $=\{(s, t):(s, t v)=(x, y)\}$, then it is easy to see that $A$ is the disjoint union

$$
A=\bigcup_{(x, y) \in A \cdot v}\left(A \cap\left[(x, y) \cdot v^{-1}\right]\right)
$$

Now

$$
\phi_{A} \cdot v=\frac{1}{|A|} \sum_{(s, t) \in A}(s, t v), \quad v \cdot \phi_{A}=\frac{1}{|A|} \sum_{(s, t) \in A}(v s, t)
$$


SO

$$
\begin{aligned}
& \left(\phi_{A} \cdot v-v \cdot \phi_{A}\right)(x, y) \\
& \quad= \begin{cases}|A|^{-1}\left|A \cap\left[(x, y) \cdot v^{-1}\right]\right| & \text { if }(x, y) \in A \cdot v \backslash v \cdot A, \\
-|A|^{-1} & \text { if }(x, y) \in v \cdot A \backslash A \cdot v, \\
|A|^{-1}\left[\left|A \cap\left[(x, y) \cdot v^{-1}\right]\right|-1\right] & \text { if }(x, y) \in A \cdot v \cap v \cdot A, \\
0 & \text { otherwise. }\end{cases}
\end{aligned}
$$

Therefore, using (3.7.1) and left cancellativity we obtain

$$
\begin{aligned}
\left\|\phi_{A} \cdot v-v \cdot \phi_{A}\right\|_{1} & =\sum_{(x, y)}\left|\left(\phi_{A} \cdot v-v \cdot \phi_{A}\right)(x, y)\right| \\
= & \frac{1}{|A|}\left[\sum_{(x, y) \in A \cdot v \backslash v \cdot A}\left|A \cap\left[(x, y) \cdot v^{-1}\right]\right|+\sum_{(x, y) \in v \cdot A \backslash A \cdot v} 1\right. \\
& \left.+\sum_{(x, y) \in A \cdot v \cap v \cdot A}\left(\left|A \cap\left[(x, y) \cdot v^{-1}\right]\right|-1\right)\right] \\
= & \frac{1}{|A|}\left[\sum_{(x, y) \in A \cdot v}\left|A \cap\left[(x, y) \cdot v^{-1}\right]\right|+|v \cdot A \backslash A \cdot v|-|A \cdot v \cap v \cdot A|\right] \\
= & \frac{1}{|A|}[|A|-|A \cdot v \cap v \cdot A|+|v \cdot A \backslash A \cdot v|]=2 \frac{|v \cdot A \backslash A \cdot v|}{|A|} .
\end{aligned}
$$

Also $\pi\left(\phi_{A}\right) v=|A|^{-1} \sum_{(s, t) \in A} s t v$, so

$$
\begin{aligned}
\left\|\pi\left(\phi_{A}\right) v-v\right\|_{1} & =\left|\left(\pi\left(\phi_{A}\right) v-v\right)(v)\right|+\sum_{x \neq v} \pi\left(\phi_{A}\right) v(x) \\
& =\left|\frac{|A \cap \nabla(v)|}{|A|}-1\right|+\frac{|A \backslash \nabla(v)|}{|A|}=2 \frac{|A \backslash \nabla(v)|}{|A|} .
\end{aligned}
$$

(Observe that left cancellativity was not used in the calculation of (3.7.3).) Let $\mathcal{F}=\{(F, \varepsilon): F \subset S$ finite, $\varepsilon>0\}$ and for each $\gamma=(F, \varepsilon) \in \mathcal{F}$, choose $A_{\gamma} \subset S \times S$ to correspond to $\gamma$ as in (*); let $m^{\gamma}=\phi_{A_{\gamma}}$. Then

$$
\left\|v \cdot m^{\gamma}-m^{\gamma} \cdot v\right\|_{1} \rightarrow 0 \quad \text { and } \quad\left\|\pi\left(m^{\gamma}\right) v-v\right\|_{1} \rightarrow 0
$$

follow from the calculations (3.7.2) and (3.7.3).

We now prove Theorem 3.2. $(1) \Rightarrow(2)$. This follows from the proofs of the two theorems found in [5]. As shown in [5], $S$ is a regular amenable semigroup so it suffices to prove that $S$ contains a unique idempotent (by regularity it has at least one). Let $E_{S}$ denote the set of idempotents in $S$ and for each $e \in E_{S}$, let $X(e)=e S \cap\left[e e^{-1}\right]$. In the proof of [5, Theorem 2] it is shown that the sets $X(e)$ partition $E_{S}$. But in the proof of [5, Theorem 1$]$ 
it is shown that when $l^{1}(S)$ is $M$-amenable, there can exist at most $M$ pairwise disjoint sets of the form $X(e)$. Thus when $l^{1}(S)$ is 1-amenable, we have $X(e)=E_{S}$ for each $e \in E_{S}$. Therefore, if $e, f \in E_{S}$, fe $=e$. As $l^{1}(S)$ is 1-amenable, so is $l^{1}\left(S^{\text {op }}\right)$ where $S^{\text {op }}$ has product $u \circ v=v u$. Hence $e=f e=e \circ f=f$.

$(2) \Rightarrow(3)$. Let $\varepsilon>0$, and let $F$ be a finite non-empty subset of $S$. From the Følner condition (FC) which characterizes amenable groups, there is a finite subset $B$ of $S$ such that

$$
|v B \cap B|>(1-\varepsilon / 2)|B| \quad(v \in F) .
$$

Let $A=\left\{\left(s, s^{-1}\right): s \in B\right\}$. Then $A \subset \nabla(v)=\left\{\left(x, x^{-1}\right): x \in S\right\}(v \in S)$, and it is readily verified that $x \mapsto\left(x, x^{-1} v\right)$ defines a bijection from $v B \cap B$ onto $v \cdot A \cap A \cdot v$. Therefore $|v \cdot A \cap A \cdot v|=|v B \cap B|>(1-\varepsilon / 2)|B|=(1-\varepsilon / 2)|A|$, whence $|v \cdot A \triangle A \cdot v|<\varepsilon|A|(v \in F)$.

$(3) \Rightarrow(4)$ is obvious.

$(4) \Rightarrow(1)$. We assume that $S$ is left subcancellative, the other case following by a symmetric argument. By Lemma 3.6, $S$ is left cancellative and $S$ satisfies condition $(*)$ of Lemma 3.7; therefore $l^{1}(S)$ is 1-amenable.

$(2) \Rightarrow(5)$ is proved by the argument given in $(2) \Rightarrow(3)$.

$(5) \Rightarrow(1)$. By Lemma 3.6, $S$ is left cancellative and condition (C) implies condition $(*)$ of Lemma 3.7; therefore $l^{1}(S)$ is 1-amenable.

REMARKS 3.8. (1) Observe that when $S$ satisfies condition (C), symmetric versions of Lemmas 3.5 and 3.6 show that $S$ is both a left and a right group, and therefore a group.

(2) Let $S$ be a discrete group. In Lemma 3.7 we saw that condition (B) naturally yields an approximate diagonal comprised of normalized characteristic functions of finite subsets $A$ of $S \times S$. Similarly, the Følner condition (FC) gives rise to a net comprised of normalized characteristic functions of finite subsets $A$ of $S$ which converges to left invariance in $l^{1}(S)$. This suggests that our conditions (A), (B), and (C) are the "correct" Følner conditions corresponding to (1-)amenability of $l^{1}(S)$.

\section{References}

[1] A. H. Clifford and G. B. Preston, The Algebraic Theory of Semigroups I, Math. Surveys 7, Amer. Math. Soc., Providence, RI, 1961.

[2] G. Corach and J. E. Galé, On amenability and geometry of spaces of bounded representations, J. London Math. Soc. (2) 59 (1999), 311-329.

[3] H. G. Dales, Banach Algebras and Automatic Continuity, Oxford Univ. Press, 2001.

[4] J. Duncan and I. Namioka, Amenability of inverse semigroups and their semigroup algebras, Proc. Roy. Soc. Edinburgh Sect. A 80 (1978), 309-321.

[5] J. Duncan and A. L. T. Paterson, Amenability for discrete convolution semigroup algebras, Math. Scand. 66 (1990), 141-146. 
[6] G. H. Esslamzadeh, Banach algebra structure and amenability of a class of matrix algebras with applications, J. Funct. Anal. 161 (1999), 364-383.

[7] E. Følner, On groups with full Banach mean values, Math. Scand. 3 (1955), 243-254.

[8] F. P. Greenleaf, Invariant Means on Topological Groups, Van Nostrand, New York, 1969.

[9] N. Grønbæk, Amenability of weighted discrete convolution algebras on cancellative semigroups, Proc. Roy. Soc. Edinburgh Sect. A 110 (1988), 351-360.

[10] - Amenability of discrete convolution algebras, the commutative case, Pacific J. Math. 143 (1990), 243-249.

[11] - Various notions of amenability, a survey of problems, in: E. Albrecht and M. Mathieu (eds.), Banach Algebras '97, de Gruyter, Berlin, 1998, 535-548.

[12] E. Hewitt and K. A. Ross, Abstract Harmonic Analysis, Springer, New York, 1963.

[13] B. E. Johnson, Cohomology in Banach algebras, Mem. Amer. Math. Soc. 127 (1972).

[14] - Approximate diagonals and cohomology of certain annihilator Banach algebras, Amer. J. Math. 94 (1972), 685-698.

[15] —, Weak amenability of group algebras, Bull. London Math. Soc. 23 (1991), $281-284$.

[16] A. T.-M. Lau and R. J. Loy, Amenability of convolution algebras, Math. Scand. 79 (1996), 283-296.

[17] V. Losert and H. Rindler, Asymptotically central functions and invariant extensions of Dirac measure, in: Probability Measures on Groups VII (Oberwolfach, 1983), Lecture Notes in Math. 1064, Springer, Berlin, 1984, 368-378.

[18] I. Namioka, Følner conditions for amenable semigroups, Math. Scand. 15 (1964), $18-28$.

[19] A. L. T. Paterson, Amenability, Amer. Math. Soc., Providence, RI, 1988.

[20] J. P. Pier, Amenable Locally Compact Groups, Wiley, New York, 1984.

[21] V. Runde, Lectures on Amenability, Lecture Notes in Math. 1774, Springer, Berlin, 2002.

[22] R. Stokke, Quasi-central bounded approximate identities in group algebras of locally compact groups, Illinois J. Math. 48 (2004), 151-170.

[23] Z. Yang, Følner numbers and Følner type conditions for amenable semigroups, ibid. 31 (1987), 496-517.

[24] Y. Zhang, Approximate complementation and its applications in studying ideals of Banach algebras, Math. Scand. 92 (2003), 301-308.

Department of Mathematics and Statistics

University of Winnipeg

515 Portage Avenue

Winnipeg, Manitoba

Canada, R3B 2E9

E-mail: r.stokke@uwinnipeg.ca

Received June 6, 2003

Revised version December 30, 2003 